\section{Hiding binary optical data with orthogonal circular polarizations}

\author{
José Luis Martínez, Ignacio Moreno, MEMBER SPIE, and \\ Felipe Mateos \\ Universidad Miguel Hernández \\ Departamento de Ciencia de Materiales \\ Óptica y Tecnología Electrónica \\ 03202 Elche, Spain \\ E-mail: i.moreno@umh.es
}

\begin{abstract}
We discuss a very simple method to encode binary data as circular polarization states with opposite helicity by means of a standard twisted nematic liquid crystal display (TN-LCD). This idea is applied to produce a steganographic imaging system, where binary data are hidden on a background with the same polarization but opposed helicity. A variant is also proposed that maintains some visible information. Experimental results obtained with a commercial TNLCD are presented. ๑ 2008 Society of Photo-Optical Instrumentation Engineers.
\end{abstract}

[DOI: 10.1117/1.2890479]

Subject terms: liquid-crystal devices (LCD); spatial light modulators; polarization encoding.

Paper 070848LR received Oct. 19, 2007; revised manuscript received Dec. 20, 2007; accepted for publication Jan. 3, 2008; published online Mar. 25, 2008.

\section{Introduction}

Light polarization has been used recently as an additional way to encode and store information. ${ }^{1,2}$ Usually, data encoding has been performed using linear polarization states with different azimuths. However, the polarization encoding is notably simplified if binary data are considered, since they can be encoded as orthogonal polarizations. For instance, the polarization encryption system in Ref. 3 uses two linear orthogonal polarization states to encode bits 0 and 1 from a two-dimensional (2-D) binary pattern. Moreover, liquid crystal displays (LCDs) provide a unique way to produce 2-D dynamically programmable systems capable of spatially modulating the state of polarization. Although this is a relatively new technique, a variety of applications have been developed, such as polarization gratings $^{4}$ and polarization diffractive elements. ${ }^{5}$ Twodimensional general polarization encoding has been demonstrated by using two $\operatorname{LCDs}^{6}$ and by using specially designed displays. ${ }^{7,8}$ In Ref. 9, a standard twisted nematic LCD (TN-LCD) was also used for linear polarization encoding by adding four external quarter wave plates (QWPs) with appropriate orientations. Here, we discuss a very simple system that uses a TN-LCD followed by a QWP to produce the two circularly polarized orthogonal states. We show that this system is useful to hide binary data (to make them invisible to the human eye), which may result in novel steganographic techniques. ${ }^{10}$

0091-3286/2008/\$25.00 @ 2008 SPIE

\section{Principle of Operation}

The proposed LCD operational mode for polarization encoding is shown in Fig. 1(a). Two LCD pixels with different addressed voltages are considered. The standard operational mode of a 90 deg TN-LCD produces two orthogonal linear states for two voltages. In the first case [bottom of Fig. 1(a)], the TN-LCD rotates the output polarization by $90 \mathrm{deg}$. In the second case [top of Fig. 1(a)], the input polarization remains unaltered since the applied voltage aligns the liquid crystal (LC) molecules. In our proposal, these two orthogonal emerging linear states are directed to a QWP oriented with its principal axis at $\pm 45 \mathrm{deg}$. Then, the QWP converts these linear states to circular states with opposite helicity, ${ }^{11}$ as indicated in Fig. 1(a). We use these L and $\mathrm{R}$ circular states to encode and hide binary data.

We employ a commercial TN-LCD from CRL-OPTO, Model XGA3-SLM, with $1024 \times 768$ pixels, a pixel size of $13 \times 10 \mu \mathrm{m}^{2}$, and a pixel pitch of $18 \times 18 \mu \mathrm{m}^{2}$. Figure $1(\mathrm{~b})$ shows the emerging polarization ellipses for the three gray levels $g=0,152$, and 255 employed in this work. These results correspond to an incident input linear polarization oriented at $20 \mathrm{deg}$ with respect to the LC director at the entrance surface of the display. We selected this angle because we found that it leads to a minimum ellipticity in the whole range of addressed gray levels. As expected, the azimuth rotates by $90 \mathrm{deg}$ from zero to the maximum addressed gray level. The ellipticity of the emerging states is
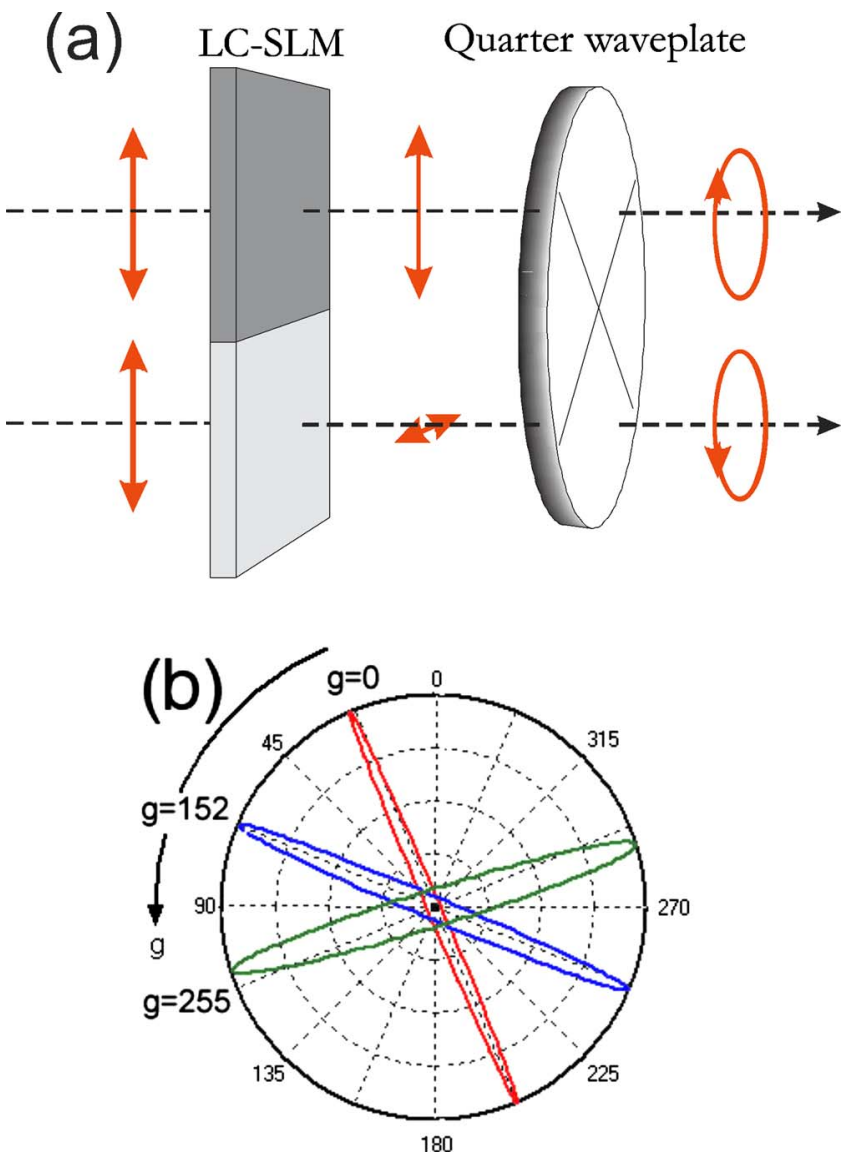

Fig. 1 (a) Scheme of the operating system. (b) Emerging polarization for gray levels $g=0,152$, and 255 . 


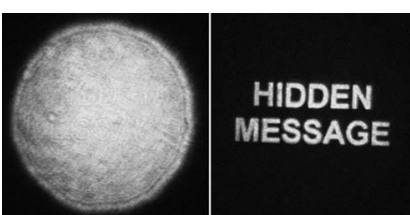

(a)

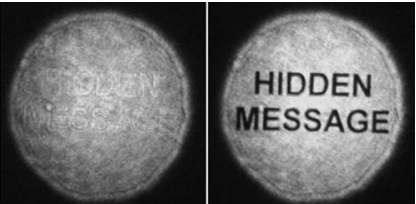

(c)

(d)

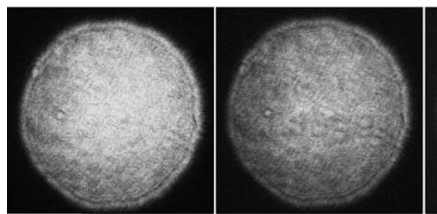

(e) (f)

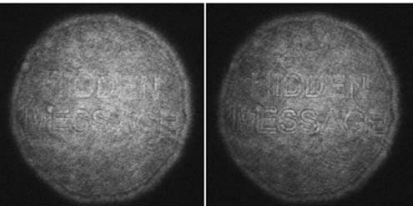

(g) (h)
Fig. 2 Captured images for binary pattern. (a) to (d) System without QWP. (e) to (h) System with QWP. No analyzer is placed behind the system in (a) and (e). Analyzer is placed behind the system oriented with relative angle $\theta=0$ deg in (b) and (f), $\theta=45$ deg in (c) and (g), and $\theta=90$ deg in (d) and (h)

not strictly zero, but it can be approximated that the display acts as a linear polarization encoder. As we show next, the two emerging states for gray levels $g=0$ and $g=255$ provide good enough results in the scheme shown in Fig. 1(a). Last, we must mention that there is additional phase term between these emerging states, which cannot be neglected in diffractive applications. ${ }^{5}$ However, since here we are interested only in intensity detection in an imaging system, this phase term becomes not relevant.

\section{Experimental Results}

The TN-LCD-QWP system can be used to approximately encode 2-D binary data as two circular states with opposite helicity. As a first example, we consider a binary pattern consisting of the words "HIDDEN MESSAGE" written with gray level $g=0$ over a background with gray level $g$ $=255$. This pattern is displayed on the LCD, and it is imaged on a CCD camera. We use a low numerical aperture lens in order to avoid any additional polarization effect. Figure 2 shows the experimental captured images in three different situations. Results in the first and second row correspond, respectively, to the system without and with the QWP behind the LCD. The images in Figs. 2(a) and 2(e) (first column) show the case without an analyzer behind the optical system. In this situation, the information is encoded in orthogonal polarizations [linear polarizations in Fig. 2(a), and L and R circular polarizations in Fig. 2(e)]. However, the absence of the analyzer produces a uniform image, and no indication about the message is detected. The analyzer very easily permits recovery of the hidden message. This is shown in Figs. 2(b)-2(d), where the analyzer is oriented with different angle $\theta$ relative to the azimuth of the emerging state for $g=0$. These figures show the captured images when the analyzer is oriented at $\theta=0 \mathrm{deg}$ [Fig. 2(b)], $\theta=45 \mathrm{deg}$ [Fig. 2(c)], and $\theta=90 \mathrm{deg}$ [Fig. 2(d)]. In Fig. 2(b), the image shows bright letters over a dark background, whereas Fig. 2(d) shows an inverted version. When the analyzer is oriented at $\theta=45 \mathrm{deg}$, the image is again uniform, since both states project to the analyzer with the same intensity. Next, consider the case when the QWP is inserted behind the LCD. Now the binary data are encoded

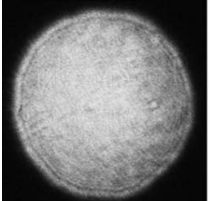

(a)

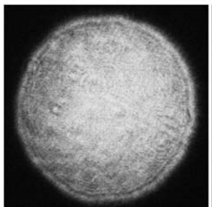

(e)

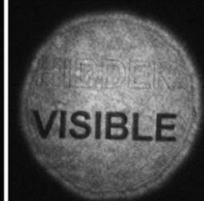

(b)

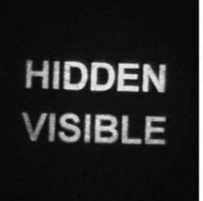

(c)

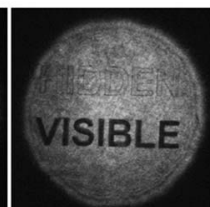

(f)

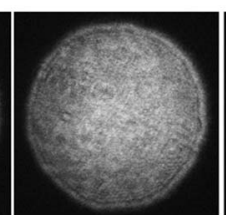

(g)

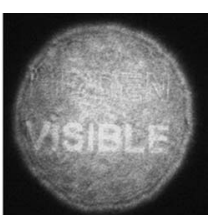

(d)
Fig. 3 Captured images for ternary pattern. (a) to (d) System without QWP. (e) to (h) System with QWP. No analyzer is placed behind the system in (a) and (e). Analyzer is placed behind the system oriented with relative angle $\theta=-45 \mathrm{deg}$ in (b) and (f), $\theta=0$ deg in (c) and $(\mathrm{g})$, and $\theta=+45 \mathrm{deg}$ in (d) and (h).

on the helicity of the circularly polarized beam. Therefore, it is not possible to retrieve the message even if the analyzer is employed. The intensity of the transmitted pattern is always one half of the incoming intensity, regardless of the orientation of the analyzer. Figures $2(\mathrm{f})-2(\mathrm{~h})$ show this situation, where the analyzer is oriented at the same angles as in the previous row. A uniform pattern is obtained in all cases, and the message remains hidden. Only a very slight effect is being produced on the edges of the letters. This edge effect has also been noticed in previous works. ${ }^{7,9}$ The fact that the information remains hidden even in the presence of the analyzer makes the system more useful for hiding information than other encodings based on linear polarization states (which become visible with a polarizer).

When using the $\mathrm{L}$ and $\mathrm{R}$ circular states, the binary data are not hidden in an actual image, but rather in constant background. In order to alleviate this limitation, a third state can be used. Gray level $g=152$ provides a third linear state with relative azimuth at $45 \mathrm{deg}$, which is not modified by the QWP since it coincides with its principal axis. This third state can be used to provide additional information that remains visible when the QWP is included in the system. Now the same system creates hidden information (using the circular states) while simultaneously maintaining some visible message (through the linear state). Figure 3 shows the experimental results. In this case, the pattern consists of the words "HIDDEN," which is encoded with gray level $g=0$, and "VISIBLE," which is encoded with gray level $g=152$, over a background encoded with gray level $g=255$. The images in the top row correspond to the system without the QWP; the QWP has been included for the images in the bottom row. As in the preceding case, we also include the experiment without the analyzer [Figs. 3(a) and 3(e)], which again shows no evidence of any encoded pattern. The results in the top row show that the three emerging states are (approximately) linear with relative orientations $\theta=0 \mathrm{deg}$ ("HIDDEN"), $\theta=45 \mathrm{deg}$ ("VISIBLE"), and $\theta=90 \mathrm{deg}$ (background). This is illustrated in Figs. $3(\mathrm{~b})-3(\mathrm{~d})$, where an analyzer is placed behind the LCD, oriented at $\theta=-45 \mathrm{deg}, 0 \mathrm{deg}$, and $+45 \mathrm{deg}$, respectively. In Fig. 3(c), the analyzer fully transmits light from the word "HIDDEN," while it fully blocks the background. The 
word "VISIBLE" appears with half the intensity. In Figs. 3 (b) and 3(d), when the analyzer is at $\pm 45 \mathrm{deg}$, the word "HIDDEN" is confused with the background, since both states project with the same intensity, while the word "VISIBLE" appears either dark or bright. More interesting is the case shown in the bottom row in Fig. 3. The addition of the QWP transforms the polarization for the word "HIDDEN" and for the background onto circular states with opposite helicity. No evidence of the word "HIDDEN" is produced when rotating the analyzer. However, the word "VISIBLE" is clearly visible as dark letters when the analyzer is at $-45 \mathrm{deg}$ [Fig. 3(f)] or as bright letters when the analyzer is at +45 deg [Fig. 3(h)]. Therefore, the hidden message is not detected, while simultaneously there is a visible message. Only when the linear polarization emerging from the word "VISIBLE" is projected onto the analyzer with half the intensity (as is the case with the circularly polarized states) is this word also confused with the background. This is the case in Fig. 3(g), when the analyzer is oriented at $0 \mathrm{deg}$.

\section{Conclusions}

In summary, we explored polarization encoding by means of a TN-LCD. First, we showed linear polarization-based encoding, which can be used to hide information to the naked human eye (in the absence of a polarizer). More interesting is the polarization encoding that uses $\mathrm{L}$ and $\mathrm{R}$ circular states, since the binary information remains hidden even when adding a polarizer. This polarization encoding is accomplished with an optical system consisting of a TNLCD followed by a QWP. Our system is simpler than other previous systems ${ }^{6-8}$ designed to generate more general polarization encodings. The same optical system can generate a third polarization state that remains linear, thus showing some additional information that remains visible. We pro- vide experimental results that demonstrate all these properties. This proposed technique could complement optical encryption techniques for the secure transmission of binary data.

\section{Acknowledgments}

This work was financed by the Ministerio de Educación y Ciencia, Spain (Ref. No. FIS2006-13037-C02-02). J. L. M. acknowledges a grant from the Instituto Alicantino de Cultura Juan Gil-Albert.

\section{References}

1. B. Javidi and T. Nomura, "Polarization encoding for optical secure systems," Opt. Eng. 39, 2439-2443 (2000).

2. X. Tan, O. Matoba, Y. Okada-Shudo, M. Ide, T. Shimura, and K. Kuroda, "Secure optical memory system with polarization encryption," Appl. Opt. 40, 2310-2315 (2001).

3. H. Y. Tu, C. J. Cheng, and M. L. Chen, "Optical image encryption based on polarization encoding by liquid crystal spatial light modulators," J. Opt. A, Pure Appl. Opt. 6, 524-528 (2004).

4. J. A. Davis, J. Adachi, C. R. Fernández-Pousa, and I. Moreno, "Polarization beamsplitters using programmable polarization diffraction gratings," Opt. Lett. 26, 587-589 (2001).

5. I. Moreno, C. Iemmi, J. Campos, and M. J. Yzuel, "Binary polarization pupil filter: theoretical analysis and experimental realization with a liquid crystal display," Opt. Commun. 264, 63-69 (2006).

6. R. L. Eriksen, P. C. Morgensen, and J. Glückstad, "Elliptical polarization encoding in two dimensions using phase-only spatial light modulators," Opt. Commun. 187, 325-336 (2001).

7. J. A. Davis, D. E. McNamara, D. M. Cottrell, and T. Sonehara, "Twodimensional polarization encoding with a phase-only liquid-crystal spatial light modulator," Appl. Opt. 39, 1549-1554 (2000).

8. K. Sueda, K. Tsubakimoto, N. Miyanaga, and M. Nakatsuka, "Control of spatial polarization by use of a liquid crystal with an optically treated alignment layer and its application to beam apodization,' Appl. Opt. 44, 3752-3758 (2005).

9. I. Moreno, J. L. Martínez, and J. A. Davis, "Two-dimensional polarization rotator using a twisted-nematic liquid crystal display," Appl. Opt. 46, 881-887 (2007).

10. S. Katzenbeiser and F. A. P. Petitcolas, Eds., Information Hiding Techniques for Steganography and Digital Watermarking, Artech House, Boston (2000).

11. E. Hetch, Optics, 4th ed., Addison-Wesley, New York (2001). 